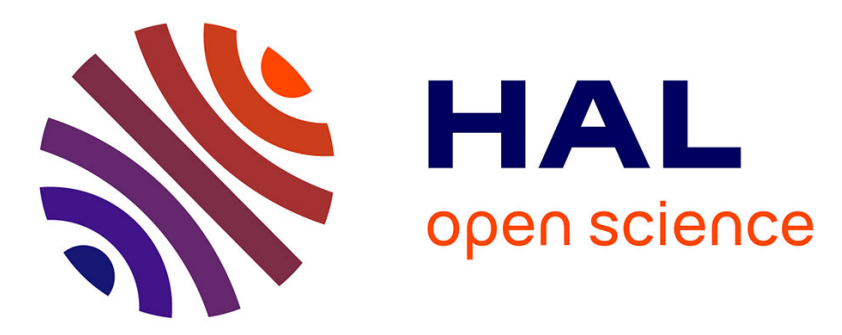

\title{
Anomalous resistive states created by light pulses in superconducting films
}

\author{
R. Buisson, R. Chicault, F. Madeore, R. Romestain
}

\section{To cite this version:}

R. Buisson, R. Chicault, F. Madeore, R. Romestain. Anomalous resistive states created by light pulses in superconducting films. Journal de Physique Lettres, 1978, 39 (9), pp.130-133. 10.1051/jphyslet:01978003909013000 . jpa-00231460

\section{HAL Id: jpa-00231460 https://hal.science/jpa-00231460}

Submitted on 1 Jan 1978

HAL is a multi-disciplinary open access archive for the deposit and dissemination of scientific research documents, whether they are published or not. The documents may come from teaching and research institutions in France or abroad, or from public or private research centers.
L'archive ouverte pluridisciplinaire HAL, est destinée au dépôt et à la diffusion de documents scientifiques de niveau recherche, publiés ou non, émanant des établissements d'enseignement et de recherche français ou étrangers, des laboratoires publics ou privés. 


\title{
ANOMALOUS RESISTIVE STATES CREATED BY LIGHT PULSES IN SUPERCONDUCTING FILMS
}

\author{
R. BUISSON, R. CHICAULT, F. MADEORE and R. ROMESTAIN \\ Laboratoire de Spectrométrie Physique $(*)$, \\ Université Scientifique et Médicale de Grenoble, B.P. 53 X, 38041 Grenoble Cedex, France
}

(Reçu le 3 février 1978, accepté le 17 mars 1978)

\begin{abstract}
Résumé. - Des expériences sur le comportement de films d'aluminium superconducteur soumis à des impulsions lumineuses ou électriques sont rapportées. Elles montrent que des états résistifs permanents peuvent être créés à des températures inférieures à la température de transition. La valeur de la résistance de ces états, inférieure à celle dans l'état normal, peut être modifiée de façon continue en changeant les conditions expérimentales.
\end{abstract}

\begin{abstract}
Experiments on the behaviour of aluminium superconducting films when submitted to light or short electric pulses are reported. They show that permanent resistive states can be created at temperatures below the transition temperature. The value of the resistance of these states, lower than the normal resistance, can be varied continuously by changing the experimental conditions.
\end{abstract}

In the last few years, the behaviour of non-equilibrium superconducting films has been the subject of many experimental and theoretical works. After the pioneer work of Testardi on laser irradiated films [1], Owen and Scalapino [2] predicted a secondorder transition in superconductors if the density of quasiparticles exceeded a critical value. This prediction has recently been verified [3] with junctions, the density of the quasi particles being modified simply by injection. The results of experiments with light pulses have shown poorer agreement $[4,5]$ with that prediction, although they show unambiguously the creation of a new state (and not a hot state) during the pulse. In this letter, we will describe results which show an anomalous behaviour of superconducting films submitted to light pulses or electrical pulses.

Two Al films deposited on a ruby substrate have been used. They are $500 \AA$ and $800 \AA$ thick. Their resistance is respectively $810 \Omega$ and $270 \Omega$ at room temperature and $325 \Omega$ and $55 \Omega$ at $4.2 \mathrm{~K}$. All the experiments are done with the film and substrate in superfluid liquid helium. The shape of these films is represented in figure 1. Transition temperature is close to $1.25 \mathrm{~K}$. Experiments performed on a granular $500 \AA$ thick $\mathrm{Al}$ film (transition temperature of about $1.6 \mathrm{~K}$ ) did not lead to any of the surprising phenomena described in this letter.

(*) Laboratoire associé au C.N.R.S.
The light pulse is generated by a dye laser pumped by a nitrogen laser. Its width is about $6 \mathrm{~ns}$ long and periodicity is typically $10 \mathrm{~Hz}$. No dependence upon wavelength was ever detected. A minimum energy of $0.5 \mu \mathrm{J}$ per pulse is needed but the effect we will describe seems to saturate for an energy larger than $5 \mu \mathrm{J}$. It is to be noted that no effect was detected with a $1 \mathrm{~mW}$ c.w. He-Ne laser.

Experimental results. - The electrical circuit is represented in figure 1 . We simultaneously monitor the value of the voltage $U$ across the film on a high frequency oscilloscope or its mean value $\bar{U}$ with a recorder. Figure 2 displays, for several values of a constant current $I$, the dependence of $\bar{R}=\bar{U} \mid I$ on the temperature of the helium bath, as measured with a carbon resistor located close to the film. In addition, the dependence of $R$ on $T$ without laser pulses is also shown for one particular value of $I$. One can see that on the high temperature side of the transition, pulses have little effect on the film (this is true whatever the value of $I$ ). One can also see (curve c) that with no irradiation there is a large hysteresis as well as sudden jumps of the resistance which can be triggered by spurious transients (when activating a near-by switch for instance). This hysteresis, which probably has a thermal origin, does not appear when the film is irradiated or submitted to electrical pulses. The dependence of the temperature corresponding to the onset of the transition on the d.c. current $I$ can be, 


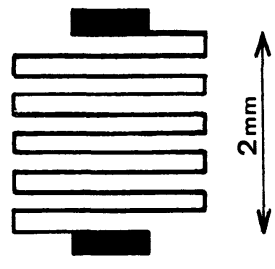

(a)

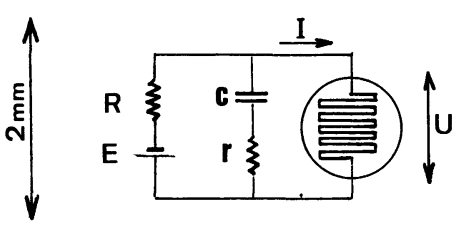

(b)
FIG. 1. - a) Shape of the superconducting film. The width of the film is $40 \mu$ and the spacing between two branches $160 \mu . b$ ) Electrical circuit used for light pulse experiments. $R$ has a high value so that the source is a current source.

at least partly, attributed to the variation of $I_{\mathrm{c}}$, the critical current, with the temperature. Temperatures $T_{\mathrm{a}}, \mathrm{T}_{\mathrm{b}}, \mathrm{T}_{\mathrm{c}}$ on figure 2 represent the temperatures at which the constant current used is equal to the critical current measured on the same film in another experiment. However, one also has to take into account the heating of the film by the Joule effect which maintains it in a normal state when decreasing the temperature and leads to the strong hysteresis mentioned.

In addition figure 2 shows the variation of the voltage $U$ across the film after a laser pulse, at a temperature $T_{2}$ and current $I=100 \mu \mathrm{A}$. $\tau_{1}$ represents the period of the pulses. One sees after a time $\tau_{2}$ a sudden jump of $U$. The value of $\tau_{2}$ increases when lowering the temperature, fluctuates severely and eventually gets longer than $\tau_{1}$ and consequently undetectable : around such a temperature the value of $\bar{U}$ gets very noisy before $U$ settles at a larger value as can be seen on the $I=100 \mu \mathrm{A}$ curve of figure 2 . The resistive state created by the laser pulse on the low temperature side of the transition is stable, and can be created by a finite number of pulses. For instance, with $I=100 \mu \mathrm{A}$ and $T_{1}$ indicated on figure 2 , we performed the following sequence with a manual triggering of the pulses :

$$
I=0 \quad \text { no pulse : }
$$

value of $\bar{R}$ represented by point A

$I=100 \mu \mathrm{A}$ no pulse :

value of $\bar{R}$ represented by point $\mathrm{A}$

$I=100 \mu \mathrm{A}$ one pulse :

value of $\bar{R}$ represented by point B

$I=100 \mu \mathrm{A}$ after several pulses :

value of $\bar{R}$ represented by point $C$

$I=100 \mu \mathrm{A}$ no pulse :

value of $\bar{R}$ represented by point $\mathrm{C}$

$I=0 \quad$ no pulse :

value of $\bar{R}$ represented by point A.

States C, B and others between C and A all have an infinite lifetime.

Using short electrical pulses of $25 \mathrm{~ns}$ width, instead of light pulses, we observe a very similar behaviour when their sign is opposite to the d.c. current flowing in the film. When their sign is the same as that of the d.c. current, they also create a stable resistive state, but no jump of $R$ can be detected on the $R(T)$ curves. As expected the electrical circuit strongly affects these phenomena because it changes the transients : the $\bar{R}$ jump is inhibited when the resistor $r$ is too large or the capacitor $C$ too small (Fig. 1).

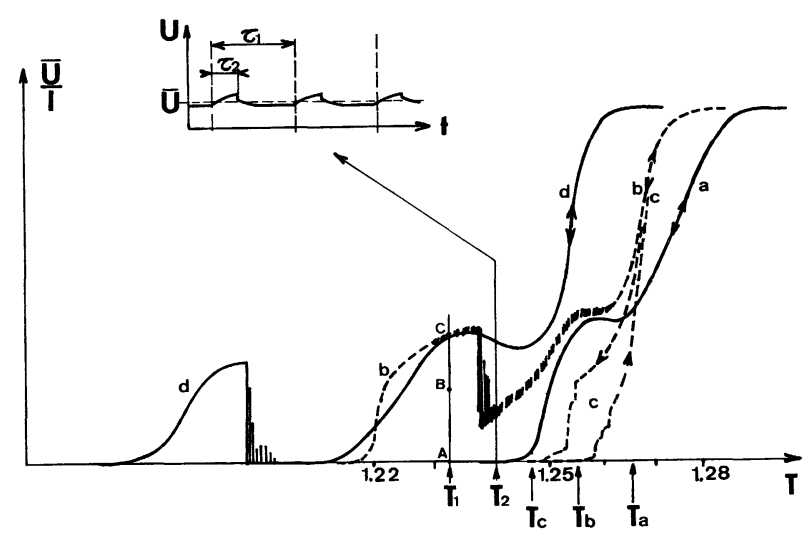

FIG. 2. - Variation of the average resistance $\bar{R}=\bar{U} / I$ of the film with temperature : a, b, d with light pulses and for $I=50,100$, $150 \mu \mathrm{A}$ respectively; c without light pulses and for $I=100 \mu \mathrm{A}$. $T_{\mathrm{a}}, T_{\mathrm{b}}, T_{\mathrm{c}}$ are the temperatures for which the critical current of the film respectively $50,100,150 \mu \mathrm{A}$. The insert shows the time variation of $U: \tau_{1}$ is the period of the pulses, $\tau_{2}$ a temperature-dependent time. States A, B, C are obtained, for $T=T_{1}$, under conditions explained in the text.

The additive effect of several such pulses is also striking. Light pulses or electrical pulses of the same sign as the d.c. current always produce an increased value of $U$ getting nearer to a situation represented by point $\mathrm{C}$ on figure 2 . On the other hand, when the sign of the electrical pulse is opposite, for certain pulse amplitudes one can reach a bistable situation where the system oscillates between two states whose representative points on figure 2 are between $\mathrm{C}$ and $\mathrm{A}$.

A further analysis of the effect of electrical pulses (without the $C$ capacitor) has shown that two parameters were important : the fall time and the amplitude. The amplitude must be larger than the critical current at the bath temperature and the fall time must not be much larger than 100 ns. However, the resistance of the metastable state created shows a maximum when increasing the amplitude further and eventually goes to zero for very large amplitudes.

The study of the $U-I$ characteristic observed at constant temperature $T<T_{\mathrm{c}}$ reveals steps which we have attributed to the successive transitions of each branch of the film [6]. The effect of the laser pulse on such characteristics has also been displayed as shown on figure 3 . The d.c. current is replaced by a square wave so that after each laser pulse the system is reset to the superconducting state by zeroing $I$. For each period and a given value of the square wave, 
one observes on the scope used on the $\mathrm{XY}$ mode, three points $\mathrm{ABC}$ :

A when $I=0, U=0$;

B when $I=I_{\mathrm{B}} \neq 0$, but $U=0$, the film being still superconducting;

C when $I=I_{\mathrm{B}} \neq 0$ and $U \neq 0$, after the laser or the electrical pulse.

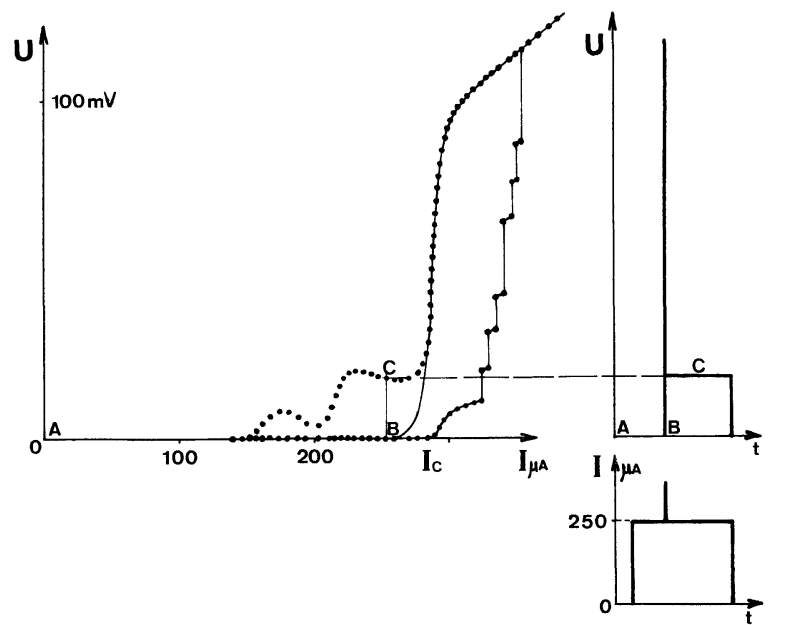

FIG. 3. $-U-I$ characteristics of a film for $T=1.2 \mathrm{~K}$. The continuous line is obtained with a triangular shape for the current. The right part corresponds to the normal state of the film. The groups of three points such as $\mathrm{ABC}$, are obtained with a pulse superimposed on a square current as illustrated on the right of the figure and as explained in the text.

Varying the value of the square wave current gives the dotted curve of figure 3 . When the bath temperature approaches $T_{\mathrm{c}}$, the curves of figure 3 are displaced toward 0 . It is easy to verify that the anomalies of these curves (the wing in the low current side) are connected to the ones observed on figure 2 .

We have studied how the resistive state depends on a partial irradiation of the film. Using a simple imaging and masking device, we were able to shine the laser pulse on only one branch of the film or even on a small part of one branch. The behaviour is strikingly different depending on whether the d.c. current is of the order of $I_{\mathrm{c}}$ (several branches may have undergone transition at this value of $I$ ) or roughly around $I_{\mathrm{c}} / 2$. In the first case irradiating a small part of a branch will either trigger the transition of the whole branch or have no effect if this branch is already resistive. In the second case the location of the irradiated part does not have any influence. Irradiating any one branch alone can lead to the same value of $U$ as obtained when irradiating the whole film provided the intensity is large enough. Whereas the first case is strictly discontinuous (branches undergo transition as a whole), the second case is continuous : every value of $\bar{R}$ between those of point $B$ and point $C$ of figure 2 can be obtained just by changing continuously the value of the pulse intensity or the amount of surface irradiated. The resistive state $\mathrm{C}$ seems to be homogeneous over the length of the film and not localized.

Discussion. - This is the first time, to our knowledge, that the observation of permanent resistive states created by laser pulses has been reported. At a given helium bath temperature, they are observed for currents well below $I_{\mathrm{c}}$ and outside the thermal hysteresis curve detected at the same temperature (Fig. 3). Warburton et al. [7] already pointed out that the curve $R$ vs $T$ obtained for $\mathrm{Sn}$ films was very sensitive to electrical filters. Without any filter they detect a wing on the low temperature side which they claim is due to electric noise inducing a partial transition of the film. This is to be compared to our reported dependence of $R$ vs $T$ (Fig. 2). But they did not observe a resistive state much below $T_{\mathrm{c}}$ after a state of zero resistance has been observed, as we did.

We have no definite explanation for these observations. In view of the homogeneous nature of the state created by the local light pulses it seems unlikely that the effects would be thermal (in the sense that a temperature variation along the film could explain the finite value of $R \neq R_{\mathrm{n}}$ observed). Against the possibility of a thermal effect, we can also point out that increasing the amplitude of the pulse, i.e. increasing the heat amount supplied to the film, reduces the resistance of the metastable state and even leaves the film in the superconducting state. This anomalous behaviour is opposite to the expected one, observed for d.c. current values corresponding to the steps of the V.I. curves of figure 3 , when a pulse puts the film in a state of higher resistance and the efficiency of the pulse increases with its amplitude. We think that an explanation has to be sought in the existence of an intermediate state, or in the possibility of several solutions of Ginsburg-Landau equations. The existence of an intermediate state in non-equilibrium superconductors has been predicted theoretically [8-10] and often put forward $[4,11]$ in the interpretation of experiments. That state would be made of small regions alternately normal and superconductive having an extension larger than $\lambda$, the coherence length, but smaller than $L$, the quasiparticle diffusion length. However, its existence is limited to temperatures lower than $0.7 T_{\mathrm{c}}$ in contradiction with this experiment. Another possible way for explaining our results could be found in the work of Tucker and Halperin [12] who have studied the influence of critical fluctuations on the resistance of superconducting films. They show that the Ginsburg-Landau equations may have several solutions and that for a given current (of sufficient value) and temperature, there can exist two stable states with different resistance. The film resistance is then due to phase slip centres which are distributed all over the film. Our observation of many different resistive states (points between $\mathrm{A}$ and $\mathrm{C}$ in 
figure 2) for the same current and temperature, seems to agree with this prediction.

Our experiments clearly show that the fall time of the pulse plays a very important role in the creation of the resistive states. Depending on the electrical circuit, that time can be made longer or shorter than the characteristic times of evolution of the off-equilibrium states. Further work will be needed to establish a connection between the various striking results reported in this letter in order to emphasize the fundamental properties of a superconducting film maintained off-equilibrium by a constant current.

Acknowledgments. - We want to acknowledge MMr. Zenati and Veler, of the L.E.T.I. (C.E.N. Grenoble), for the preparation of the high quality films used in these experiments.

\section{References}

[1] Testardi, L. R., Phys. Rev. B 4 (1971) 2189.

[2] OWen, C. S. and SCAlaPino, D. J., Phys. Rev. Lett. 28 (1972) 1559.

[3] Fuchs, J., Epperlein, P. W., Welte, M. and Eisenmenger, W., Phys. Rev. Lett. 38 (1977) 919.

[4] Sai-Halasz, G. A., Chi, C. C., Denenstein, A. and LangenBERG, D. N., Phys. Rev. Lett. 33 (1974) 215.

[5] Golovashkin, A. I., Mitsen, K. V. and Motulevich, G. P., Sov. Phys. J.E.T.P. 41 (1975) 701.

[6] To be published.
[7] Warburton, R. J., Patton, B. R., Webb, W. W. and Wilkins, J. W., Physica 55 (1971) 324.

[8] Chang, J. J. and Scalapino, D. J., Phys. Rev. B 10 (1974) 4047.

[9] BARU, V. G. and Sukhanov, A. A., J.E.T.P. Lett. 21 (1975) 93.

[10] Scalapino, D. J. and Huberman, B. A., Phys. Rev. Lett. 39 (1977) 1365.

[11] Kaplan, S. B., Kirtley, J. R. and Langenberg, D. N., Phys. Rev. Lett. 39 (1977) 291.

[12] TUCKer, J. R. and HalPerin, B. I., Phys. Rev. B 3 (1971) 3768. 